\title{
Angiogenic Potential of Human Neonatal Foreskin Stromal Cells in the Chick Embryo Chorioallantoic Membrane Model
}

\author{
Radhakrishnan Vishnubalaji, ${ }^{1}$ Muhammad Atteya, ${ }^{1,2}$ May Al-Nbaheen,, \\ Richard O. C. Oreffo, ${ }^{1,4}$ Abdullah Aldahmash, ${ }^{1,5}$ and Nehad M. Alajez ${ }^{1}$ \\ ${ }^{1}$ Stem Cell Unit, Department of Anatomy, College of Medicine, King Saud University, Riyadh 11461, Saudi Arabia \\ ${ }^{2}$ Histology Department, Faculty of Medicine, Cairo University, Cairo, Egypt \\ ${ }^{3}$ Saudi Electronic University, Saudi Arabia \\ ${ }^{4}$ Bone and Joint Research Group, Centre for Human Development Stem Cells and Regeneration, Human Development and Health, \\ Institute of Developmental Science, University of Southampton, Southampton, UK \\ ${ }^{5}$ KMEB, Department of Endocrinology, University of Southern Denmark, Odense, Denmark
}

Correspondence should be addressed to Radhakrishnan Vishnubalaji; vishnubalaji.lr@gmail.com and Nehad M. Alajez; nalajez@ksu.edu.sa

Received 5 May 2015; Revised 9 June 2015; Accepted 10 June 2015

Academic Editor: Tong-Chuan He

Copyright (C) 2015 Radhakrishnan Vishnubalaji et al. This is an open access article distributed under the Creative Commons Attribution License, which permits unrestricted use, distribution, and reproduction in any medium, provided the original work is properly cited.

\begin{abstract}
Several studies have demonstrated the multipotentiality of human neonatal foreskin stromal cells (hNSSCs) as being able to differentiate into adipocytes and osteoblasts and potentially other cell types. Recently, we demonstrated that hNSSCs play a role during in vitro angiogenesis and appear to possess a capacity to differentiate into endothelial-like cells; however, their angiogenic potential within an ex vivo environment remains unclear. Current study shows hNSSCs to display significant migration potential in the undifferentiated state and high responsiveness in the in vitro wound healing scratch assay. When hNSSCs were seeded onto the top of the CAM, human von Willebrand factor (hVWF), CD31, smooth muscle actin (SMA), and factor XIIIa positive cells were observed in the chick endothelium. CAMs transplanted with endothelial-differentiated hNSSCs displayed a higher number of blood vessels containing hNSSCs compared to CAMs transplanted with undifferentiated hNSSCs. Interestingly, undifferentiated hNSSCs showed a propensity to differentiate towards ectoderm with indication of epidermal formation with cells positive for CD1a, CK5/6, CK19, FXIIIa, and S-100 cells, which warrant further investigation. Our findings imply a potential angiogenic role for hNSSCs ex vivo in the differentiated and undifferentiated state, with potential contribution to blood vessel formation and potential application in tissue regeneration and vascularization.
\end{abstract}

\section{Introduction}

Angiogenesis is a multifaceted process that involves endothelial cell proliferation, migration and differentiation, extracellular matrix (ECM) remodelling, and the functional development of new blood vessels from preexisting vasculature. The exploration of angiogenesis offers new approaches to understanding the mechanisms underlying vascular disease and to aid in regeneration. Furthermore, stem cell transplantation has emerged in the last few years as a potential therapy for several diseases, given the potential of stem cells to differentiate into multiple lineages and the prospect that they may offer trophic support for cell survival, tissue restoration, and functional improvement [1-3].

Mesenchymal stem cells or multipotent stromal cells (MSCs) are nonhematopoietic stem cells with extensive selfrenewal and multilineage differentiation potential [4-7]. In our previous study, hNSSCs were shown to express thirtythree CD markers including known stromal cell-associated as well as several novel markers [6]. Moreover, these cells could be induced to differentiate into cells expressing endothelial markers and to form densely packed large diameter tubules during in vitro angiogenesis assay $[5,8]$. However, the angiogenic capacity of hNSSCs ex vivo remains unclear. 
Autologous stem cell transplantation has been employed to aid therapeutic angiogenesis in various diseases, including ischemic cardiac and limb disease and connective tissue disorders. Nonetheless, there is substantial heterogeneity in the system of recruitment, collection, and storage of autologous clinical grade source [9]. Our preliminary studies using neonatal foreskin showed promising results indicating that hNSSCs could be an alternative potential source for cell based angiogenesis [6, 8]. Thus, improved understanding of the cellular mechanisms of hNSSCs vasculogenesis and angiogenesis could offer new therapeutic approaches for hNSSCs.

The current study has examined the angiogenic potential of hNSSCs in an ex vivo angiogenic assay. The chick chorioallantoic membrane (CAM) assay offers excellent nutrient supply given the dense capillary network and preexisting vasculature providing a robust angiogenic ex vivo model to assay cells, scaffolds, and growth factors including a foundation of vessels that expand into implanted hNSSCs [10-13]. The assay is robust and economical, and, critically, the chick immune system is not fully developed allowing analysis of cells and materials without issues of immune rejection. Furthermore, the model has been used to investigate the efficiency and mechanisms of action of pro- and antiangiogenic natural and synthetic materials $[10,14,15]$. Thus we have used the CAM model to investigate the functional potential of hNSSCs to contribute to angiogenesis in an ex vivo environment.

\section{Methodology}

2.1. Ethics Statement. The use of human specimens in current study was approved by the Institutional Review Board at King Saud University College of Medicine (10-2815-IRB). The embryonic chicken chorioallantoic membrane assay was carried out at the University of Southampton according to Home Office Approval UK under the Project license-PPL $30 / 2762$.

2.2. Isolation and Culture of hNSSCs. hNSSCs were isolated and cultured in accordance with our previously published protocols $[6,8]$. In brief, cells were isolated by explant organ culture to establish outgrowth cell culture (Figure 1(a)). Newborn foreskins were received from voluntary circumcisions with informed consent. Tissues were washed and the epidermis was removed followed by the dermis. Tissues were placed in culture dishes with the epidermis layer facing upwards and the dermis area in contact with the plastic surface with a droplet of culture medium. Cultures were maintained at $37^{\circ} \mathrm{C}$ and $5 \% \mathrm{CO}_{2}$ in a humidified environment. Additional media were added following cell attachment and culture was maintained for 7 days or until outgrowths of fibroblast-like spindle shaped cells were observed. At 70-80\% confluency, cells were trypsinized and residual tissues were removed. The culture medium consisted of Dulbecco's Modified Eagle Medium (DMEM) supplemented with D-glucose $4500 \mathrm{mg} / \mathrm{L}$, $4 \mathrm{mM}$ L-Glutamine, and $110 \mathrm{mg} / \mathrm{L}$ Sodium Pyruvate, 10\% Fetal Bovine Serum (FBS), 1x penicillin-streptomycin (Penstrep), and nonessential amino acids (all purchased from Gibco-Invitrogen, USA).
2.3. Aldefluor Assay. The Aldefluor kit (Stem Cell Technologies, Vancouver, BC, Canada) was used to determine the percentage of cells with high Aldehyde Dehydrogenase (ALDH) enzymatic activity. Briefly, $10^{6}$ cells were resuspended in Aldefluor assay buffer containing ALDH substrate as recommended by the manufacturer. As a negative control for all samples, an aliquot of "Aldefluor-exposed" cells was immediately quenched using an ALDH inhibitor, diethylaminobenzaldehyde (DEAB). After 30 minutes of incubation at $37^{\circ} \mathrm{C}$, the cells were centrifuged and resuspended in $500 \mu \mathrm{L}$ Aldefluor buffer and analyzed using BD FACS Calibur flow cytometer (BD Biosciences). Aldefluor staining was detected within the green fluorescence channel FL1. Samples treated with the inhibitor DEAB (+DEAB) were used as controls to establish the gates defining the $\mathrm{ALDH}^{+}$region. Cell Quest Pro Software Version 3.3 (BD Biosciences) was used to analyze the data.

2.4. Transwell Migration Assay. The hNSSCs transwell migration assay was performed as we described before [16]. Briefly, transwell membrane ( 8 um pore; BD Falcon) inserts were used in the study. The undifferentiated cells were trypsinized, washed, and resuspended in medium without FBS. To the lower wells of the chambers, migration-inducing medium (with $1 \%$ and $15 \%$ FBS) was added. The upper wells were filled with serum-free medium containing cells $\left(1 \times 10^{5}\right.$ cells per well). The chamber was incubated at $37^{\circ} \mathrm{C}$ and $5 \%$ $\mathrm{CO}_{2}$ in a humidified environment. After $24 \mathrm{~h}$, assays were stopped with removal of the medium from the upper wells and careful removal of the filters. Filters were fixed using 4\% paraformaldehyde and stained with eosin. Assessment of completed transmigration was performed by microscopy, and random fields were examined (four fields per filter) for the presence of cells on the lower membrane side only (Carl Zeiss-Axio Observer.1 equipped with a digital camera (Axiocam MRc5)). The number of migrated cells was counted using Image-Pro Plus software (Media Cybernetics).

2.5. The hNSSCs Real-Time Migration. Real-time analysis of cell migration was executed using the xCELLigence RTCA DP system (ACEA Biosciences, San Diego, CA). 40,000 cells were seeded per well in a 16-well microelectronic sensing, two-chamber transwell plates (CIM-plates) containing the respective serum conditions. Medium containing 15\% serum (chemoattractant) and $1 \%$ serum (control) was added to the bottom wells. Migration of cells is measured as a result of the interaction of cells with the electrodes on the bottom surface of top chamber and represented as a change in cell index (CI), an arbitrary unit derived from the relative change in electrical impedance across microelectronic sensor arrays. The electrical impedance was captured every $15 \mathrm{~min}$ for an experimental duration of $\sim 68 \mathrm{~h}$. The rate of migration is expressed as the CI or the change in electrical impedance at each time-point. Values are expressed as the \pm SEM of the 8 replica wells from three independent experiments.

2.6. In Vitro Scratch Assay (Wound Healing Model). For the assessment of cell migration, confluent undifferentiated hNSSCs maintained in standard medium were wounded with 
Day 3
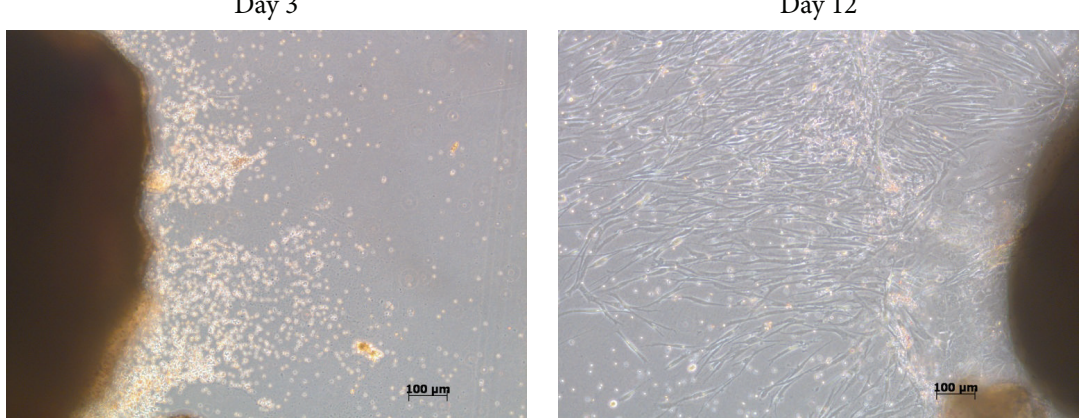

(a)
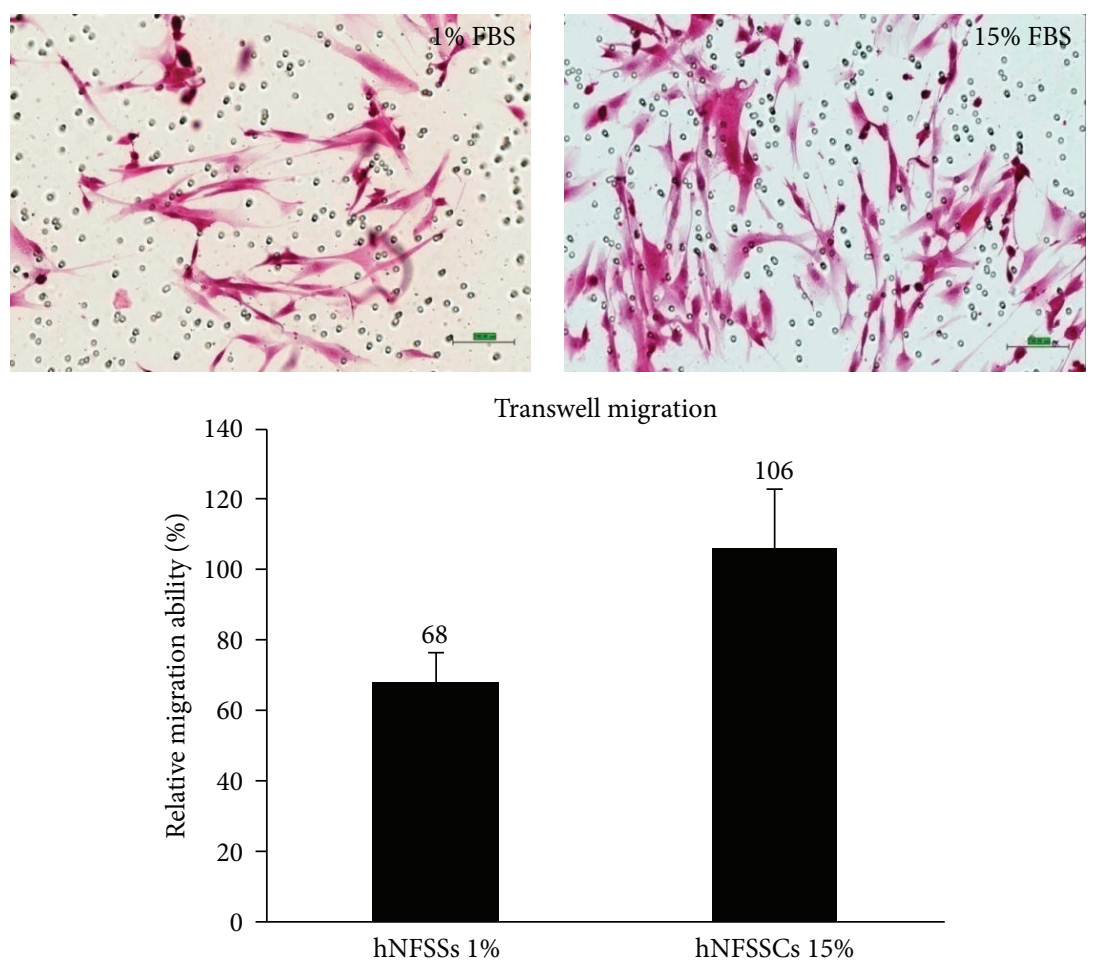

(b)

hSSCs real-time migration
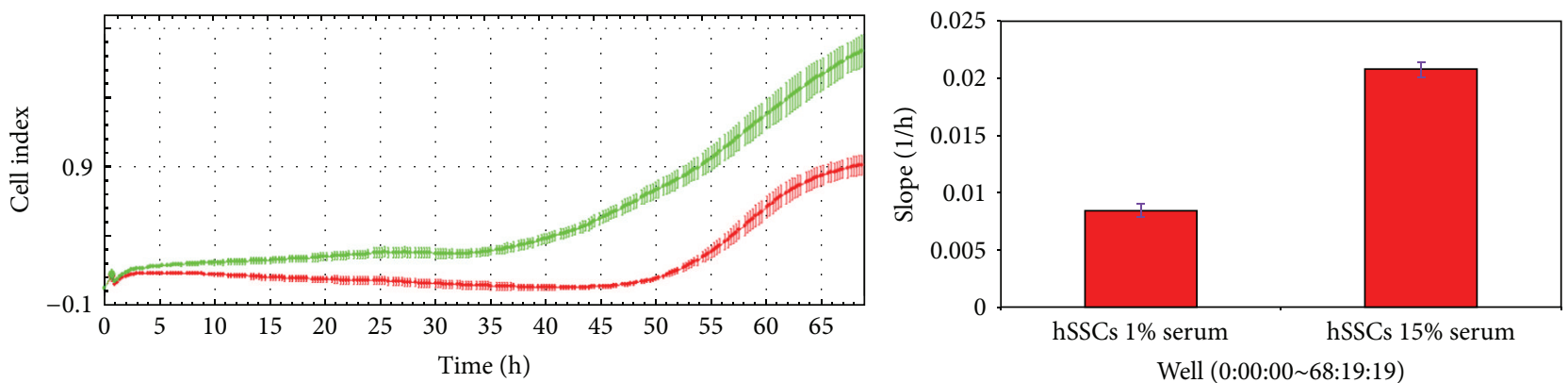

$1 \%$ serum

$15 \%$ serum

(c)

FIgURE 1: Continued. 
Scratch wound healing assay
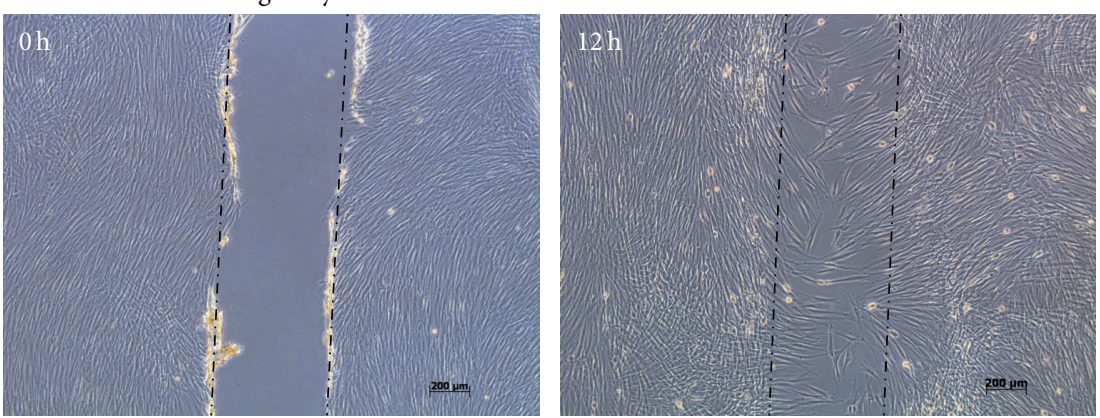

(d)
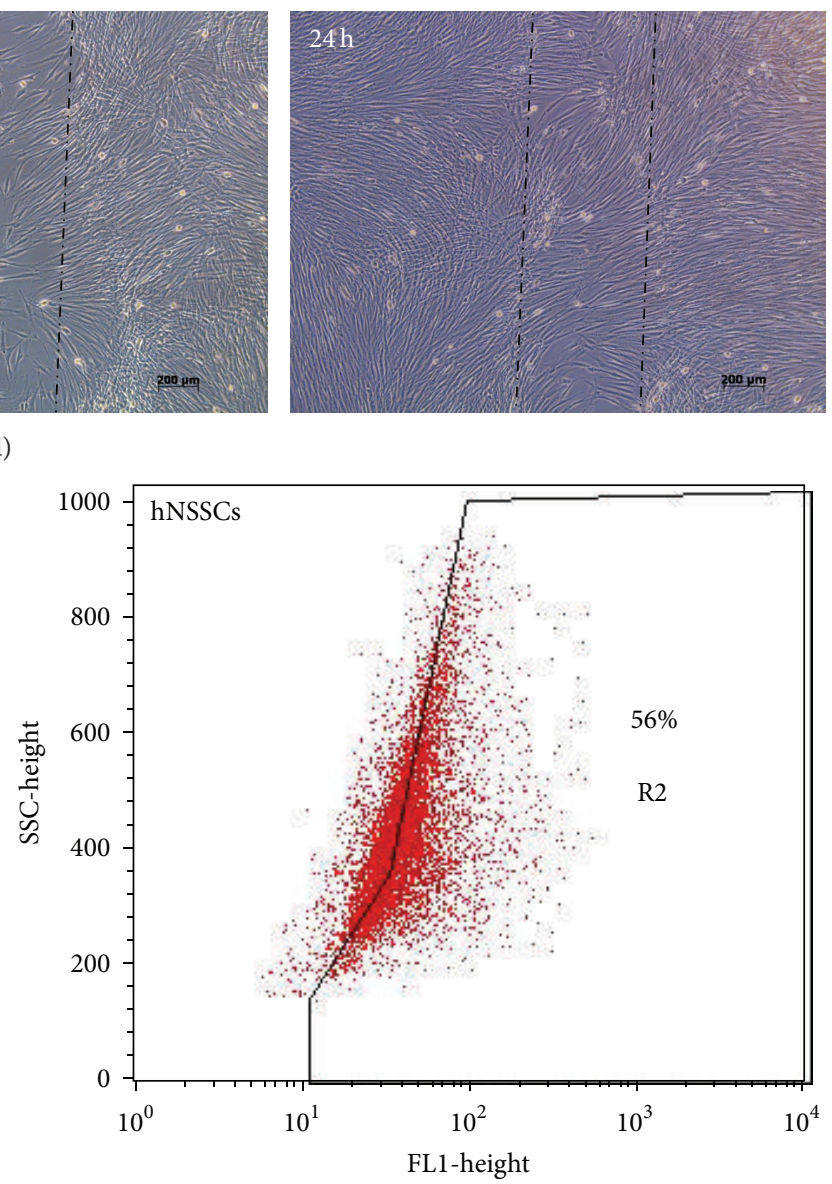

(e)

FIGURE 1: Explant organ culture system of neonatal foreskin. (a) Cell migration from skin tissue after 3 days (left); fibroblast-like cells can be observed migrating and sprouting out from tissue after 12 days (right). (b) A transwell migration assay where cells migrated to the lower chamber visualized by Eosin staining. The transwell migration assay was repeated and the total number of cells that migrated was quantified to determine the relative numbers of migrated cells (b, lower panel). Data are presented as mean \pm S.D. The experiment was run in triplicate. (c) The real time migration was executed using the xCELLigence RTCA DP device. Cells were seeded per well in 16-well microelectronic sensing, two-chamber transwell plates (CIM-plates). The electrical impedance was captured every $15 \mathrm{~min}$ for an experimental duration of $\sim 68 \mathrm{~h}$. The rate of migration is expressed as the CI or the change in electrical impedance at each time-point (c, left). Values are expressed as the \pm SEM of the 8 replica wells from three independent experiments (c, right). (d) Measurement of undifferentiated hNSSCs cell migration by in vitro wound healing scratch assay. The migration ability was assessed $12 \mathrm{~h}$ and $24 \mathrm{~h}$ from injury. The figure shows the migration of undifferentiated cells immediately after the scratch $0 \mathrm{~h}, 12 \mathrm{~h}$, and $24 \mathrm{~h}(\mathrm{Bar}=100 \mu \mathrm{m})$. (e) ALDH activity of hNSSCs was measured using the Aldefluor assay and FACS analysis. Cells incubated with specific inhibitor of ALDH, DEAB, were used to establish the baseline fluorescence of these cells and to define the Aldefluor positive region.

a plastic micropipette tip (yellow tip; 20-200 $\mu \mathrm{L}$ ). After washing, the medium was replaced by fresh medium. Photographs of the wounded area were taken after 12 and $24 \mathrm{hrs}$ using phase-contrast microscopy. For evaluation of wound closure, four randomly selected points along each wound area were marked, and the horizontal distance of migrating cells from the initial wound was measured (Carl Zeiss-Axio Observer.1 equipped with a digital camera (Axiocam MRc5)).

2.7. Endothelial Differentiation. hNSSCs were differentiated as previously detailed [5]. At 70-90\% confluency medium was replaced with endothelial induction medium (Medium
199 with Earle's salt with L-Glutamine (PAA; Cat. Number E15-834) + 10\% Fetal Calf Serum (FCS), 1\% Pen-strep, $2 \mathrm{ng} / \mathrm{mL}$ VEGF (R\&D systems, USA), and ECGS/H $3 \mathrm{mg} / \mathrm{mL}$ protein with $22.5 \mathrm{mg} / \mathrm{mL}$ Heparin (endothelial cell growth supplements (ECGS), Promocell, UK) for 7 days). The medium was changed every 2 days.

2.8. Organotypic Culture. hNSSCs were cultured on the CAM as previously detailed allowing the analysis of hNSSCs in an ex vivo culture (Figure 3) [12]. After Trypsinization, cell pellets were resuspended in $10 \mathrm{~mL}$ culture medium (control cells in routine culture medium, induced cells in endothelial 
induction medium). Cell count was performed to give a cell density of between $3 \times 10^{5}$ and $5 \times 10^{5} / \mathrm{mL}$. One $\mathrm{mL}$ of cell suspension was added per falcon tube, centrifuged at $400 \mathrm{~g}$ for 10 minutes and incubated at $37^{\circ} \mathrm{C}$, supplemented with $5 \% \mathrm{CO}_{2}$. After 2 days, pellets formed were cultured for 2 more days on organotypic culture until cell attached to the confetti (Figures 3(a) and 3(b)) (hydrophilic PTFE (polytetrafluoroethylene) membranes ( $0.4 \mathrm{~mm}$ pore size); Millipore; UK).

2.9. Ex Vivo Angiogenesis Using the Chick Chorioallantoic Membrane Assay. The chick chorioallantoic membrane (CAM) was exposed by cutting a window $\left(2 \mathrm{~cm}^{2}\right)$ on one side of 10-day-old specific pathogen-free chicken egg (Figure 3(c)). Confetti organotypic culture was placed on the CAM. The window in the shell was sealed with adhesive tape and the egg incubated for 10 days. Representative CAMs from each group (untreated and treated) were imaged using a dissecting microscope (10x) and counted (Figures 3(d), $3(\mathrm{e}), 3(\mathrm{f})$, and $3(\mathrm{~g}))$. The number of fine distinct blood vessel branch points in the area of the confetti was counted [17]. As angiogenesis is categorized by the sprouting of new vessels from preexisting vessels in response to hNSSCs, thus counting blood vessel branching points is a functional quantitative means of determining angiogenic index. At least 5 embryos were used per group. Data were assessed in terms of average number of branching points per group \pm standard deviation.

2.10. Histological and Immunohistochemical Analysis of CAM. Representative CAMs from each group were excised and fixed in formalin and subsequently dehydrated through a series of graded alcohols and embedded in low-melting point paraffin using an automated Shandon Citadel 2000. Tissue sections $6 \mu \mathrm{m}$ thick were cut from across the CAM contacting confetti and stained for the light green and alcian blue counter stain, followed by staining with CD 31 (CD 31; 1:50; Proteintech Europe) and vWF (1:200; Dako) to determine angiogenesis. For further analysis, formalinfixed paraffin-embedded CAM were stained according to the manufacturer's staining protocol on a Bondmax fully automated IHC and ISH staining system (Leica Microsystems $\mathrm{GmbH}$, Germany) and stained with haematoxylin and eosin (H\&E). Antibodies were from Novocastra (Leica Biosystems) ready to use except CD1a (Clone MTB1; PA0235), CK19 (Clone b170; PA0799), FXIIIa (Clone E980.1; PA0449), S100 (Polyclonal; PA0900), SMA (alpha sm-1; PA0943), and CK5/6 (Clone D5 \& 16B4; 356 M) which were from Cell Marque and were used according to manufacturer's standard protocols. Slides were digitized using high-resolution wholeslide digital ScanScope scanner (Aperio Technologies, Inc.). Mouse monoclonal (EMR8-5; ab70328; Abcam) to HLA Class $1 \mathrm{ABC}$ was used to identify the human cells in CAM. All the staining was performed with respective positive control tissues according to the antibody manufacturer instructions and without primary antibodies; the secondary antibody staining was considered as experimental negative control (Supplementary Figures 1 and 2 in Supplementary Material available online at http://dx.doi.org/10.1155/2015/257019).
The digital slide images were viewed and analyzed using Aperio's ImageScope software (Aperio Technologies, Vista, CA, USA).

2.11. Statistical Analysis. All measurements were calculated as mean percentage \pm standard deviation. Statistical analysis was performed using Microsoft Excel 2007 software. Differences among groups were determined using $t$-test and statistical differences were considered to be significant if $P \leq 0.05$. All experiments were performed a minimum of three times.

\section{Results}

3.1. hNSSCs Possess High Migration Potential In Vitro and Are Enriched in $A L D H^{+}$Cells. The outgrowth of fibroblastlike spindle shaped cells was observed in neonatal foreskin explants organ cultures as early as three days after culture with extensive cell outgrowth observed by day 12 (Figure 1(a)). We previously characterized the phenotype, colony forming unit, population doubling, and differentiation potential of hNSSCs in comparison with conventional bone marrow-derived MSCs $[6,8]$. We subsequently examined the migration potential of hNSSCs using a manual transwell and automated realtime migration system. hNSSCs were seeded in the upper chamber of a transwell migration system $(8.0 \mu \mathrm{m}$ pore size $)$ in the manual assay and we followed the manufacture protocol in automated system, and media (DMEM) supplemented with 15\% FBS were used as an attractant. As illustrated in Figures 1(b) and 1(c), naive hNSSCs displayed high migration potential in vitro. Similarly, hNSSCs also exhibited enhanced migration potential when assessed using an in vitro wound healing assay at both 12 and $24 \mathrm{hrs}$ after scratch (Figure 1(d)). The presence of potential stem cells within hNSSC cultures was assessed using the Aldefluor assay, which has been utilized to examine the frequency of stem cells in a number of cell types which typically display high ALDH activity $[18,19]$. The ALDH inhibitor (DEAB) was used to ensure specificity of the assay and appropriate FACS analysis. FACS analysis indicated the frequency of ALDH+ cells in hNSSCs to be around $52 \pm 0.9 \%$ (Figure $1(\mathrm{e})$ ). These results suggest high frequency of undifferentiated progenitor/stem cells within the hNSSC cultures.

\subsection{Differentiated and Undifferentiated hNSSCs Displayed} Different Morphological Changes When Cultured on Different Surfaces. Interestingly, undifferentiated and endothelialdifferentiated hNSSCs displayed distinct morphology upon placement on treated and nontreated tissue culture plastic surface. Undifferentiated hNSSCs maintained their spindle shape when cultured on treated and nontreated tissue cultures surfaces (Figures 2(a) and 2(c)), while those cells exhibited modest tube formation potential when plated on matrigel (Figures 2(e)). On the other hand, endothelial-differentiated cells were observed to form matrix-rich tubular-like structure on tissue culture treated plastic surface (Figure 2(b)) and to form spheres when cultured on nontissue culture treated surface (Figure 2(d)). Most notably, differentiated hNSSCs formed tight tubular-like structure when plated on matrigel (Figure 2(f)). 


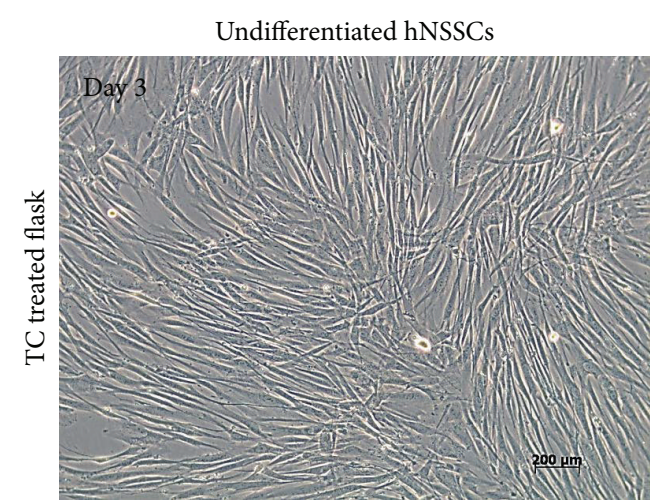

(a)

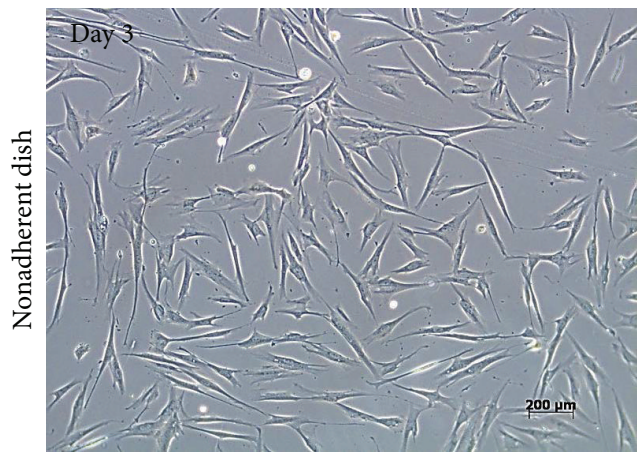

(c)

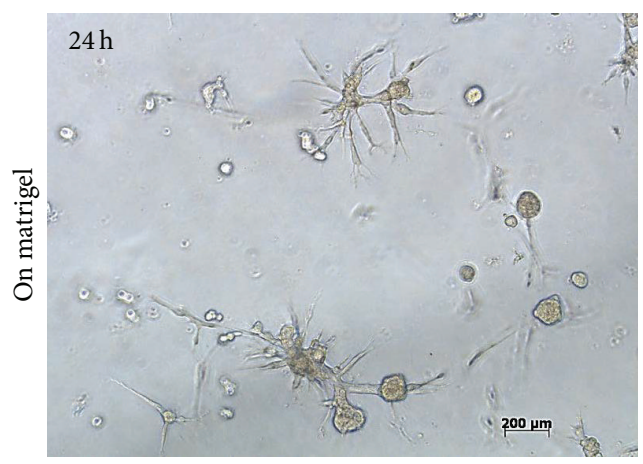

(e)

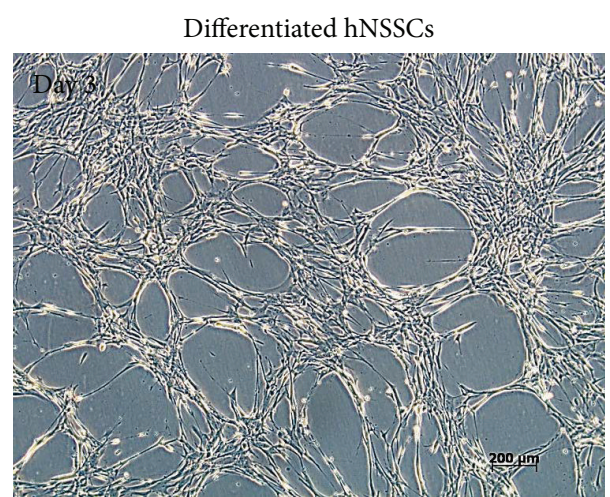

(b)

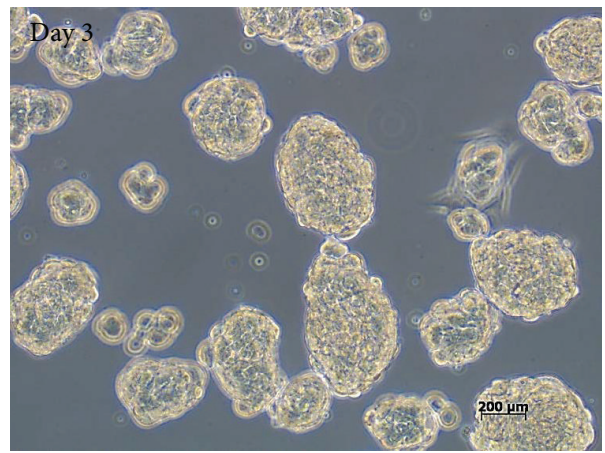

(d)

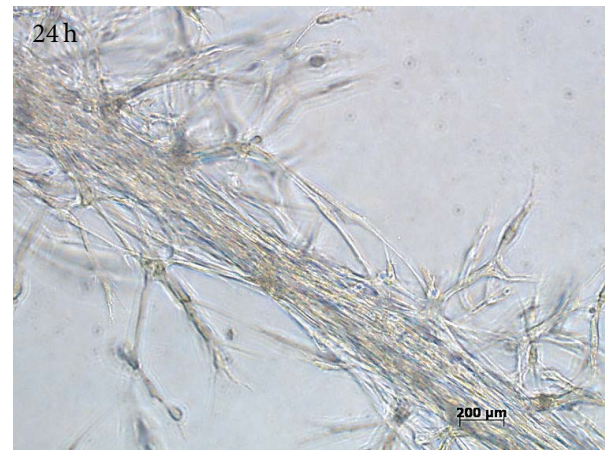

(f)

FIGURE 2: Undifferentiated and differentiated hNSSCs exhibit different morphological changes when cultured on different surfaces. Undifferentiated and differentiated hNSSCs were cultured on tissue culture-treated (a and b), or untreated (c and d) or on matrigel (e and f). Morphological changes were subsequently observed and imaged (Bar $=200 \mu \mathrm{m})$.

3.3. hNSSCs Contribute to Ex Vivo Vasculogenesis. We have previously shown the ability of hNSSCs to differentiate into endothelial-like cells in vitro [8]; however, the ability of hNSSCs to participate in neovasculature ex vivo has not been addressed to date. To assess hNSSCs ex vivo differentiation potential, the pellets of hNSSCs undifferentiated or differentiated under endothelial-induction conditions were cultured on PTFE confetti for 2 days as shown in schematic diagram (Figures 3(a) and 3(b)). Subsequently, cell pellets with confetti were transplanted onto CAMs by making $2 \mathrm{~cm}^{2}$ window on ten-day-old chick embryos (Figure 3(c)); after a further ten days of growth, CAMs were imaged and assessed for vascular formation (Figures 3(d)-3(f)). CAMs transplanted with differentiated and undifferentiated hNSSCs exhibited significant blood vessel formation. The number of vessel branching points was significantly $(P \leq 0.05)$ higher in CAMs transplanted with differentiated hNSSCs $(190 \pm$ branching points) compared to CAMs transplanted with undifferentiated hNSSCs (110 \pm branching points, Figure 3(g)). Control CAM cultures displayed the lowest number of branching points (70 \pm branching points). Similarly, immunohistochemical staining revealed larger number of blood vessels in CAMs transplanted with differentiated hNSSCs compared to CAMs transplanted with undifferentiated hNSSCs 


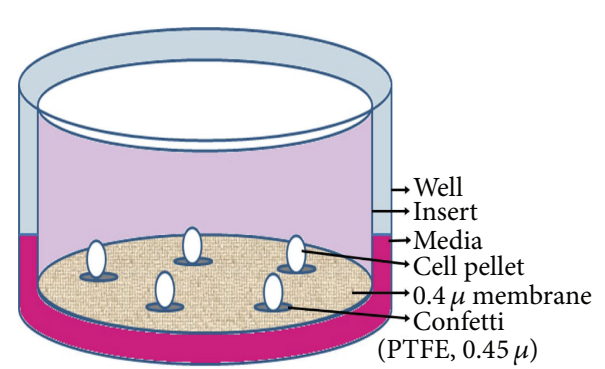

(a)

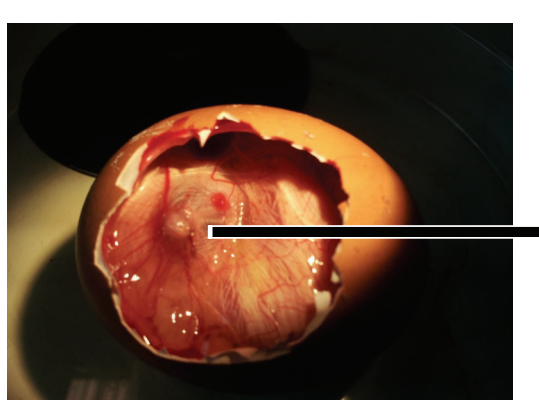

(d)

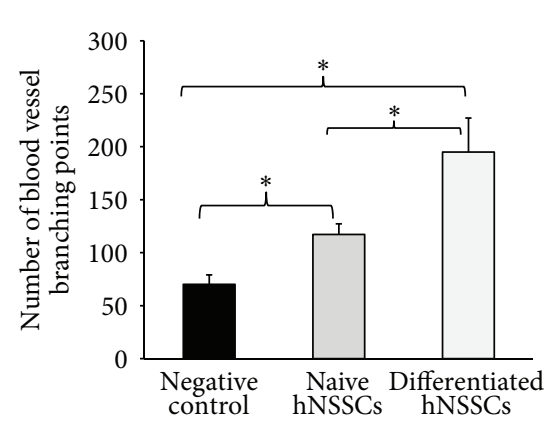

(g)

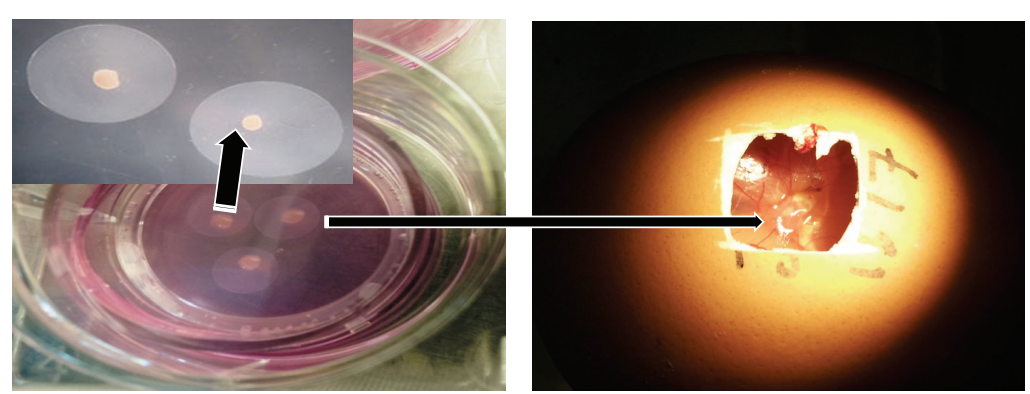

(b)

(c)

Differentiated hNSSCs

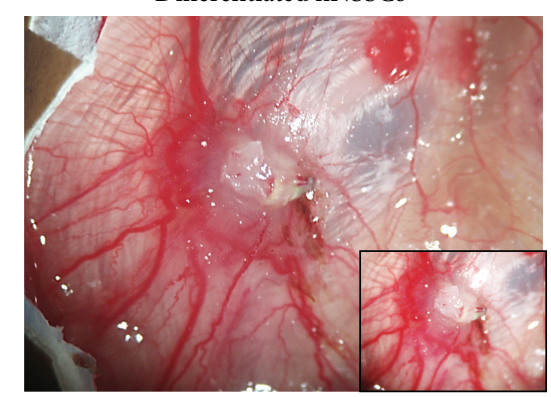

(f)

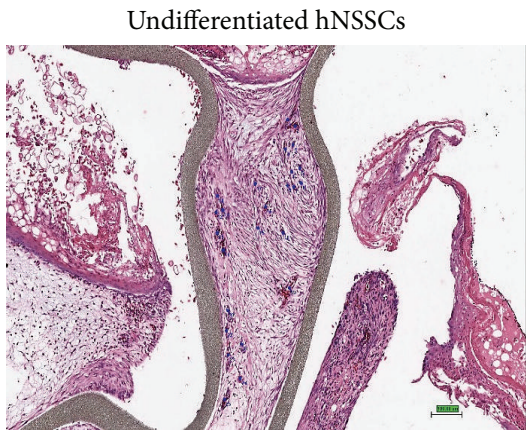

(h)

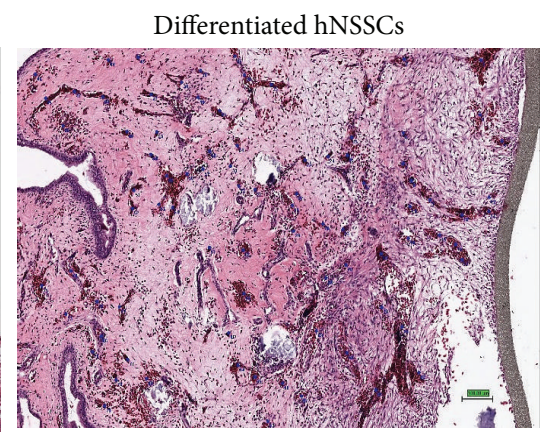

(i)

FIGURE 3: hNSSCs contribute to neovasculature using ex vivo CAM model. (a) Schematic illustration of the organotypic culture system (liquid-air interface). hNSSCs pellets were placed on the top of a confetti that is in contact with the membrane on the insert. The media diffuse across the insert membrane and confetti to reach the pellet. Microscopic aspect of chick chorioallantoic membrane (CAM) and organotypic cultured hNSSCs with confetti grafted to it. (b) hNSSCs pellet placed on top of a piece of confetti that is in contact with the membrane on the insert. (c) Window $\left(2 \mathrm{~cm}^{2}\right)$ made in the egg shell of a 10-day-old chick embryo with organotypic culture transplanted. (d) After 10 days, implantation shell was opened and blood vessel points were observed; (e and f) note the wheel-spoke pattern of CAM blood vessels around the graft (undifferentiated and differentiated). Stimulation of angiogenesis in CAM by undifferentiated or differentiated hNSSCs. The expansion of new blood vessels was determined by counting branch points after 10 days of cell transplantation. Sprouting and branching vessels are prominent in the CAM transplanted by differentiated hNSSCs compared to the undifferentiated hNSSCs transplantation. (g) Quantification of new blood vessels formed by naive (undifferentiated) and differentiated hNSSCs compared to negative control (confetti without cell transplantation) $(n=5) .{ }^{*} P<0.05$. (h, i) Paraffin sections of both undifferentiated and differentiated hNSSCs transplanted CAM were stained by haematoxylin and eosin stain to show the vascular density in CAM; blood vessels were counted again (blue colour points indicate vasculature) with Aperio's ImageScope software (Aperio Technologies, Vista, CA, USA) $($ Bar $=100 \mu \mathrm{m})$.

(Figures 3(i) and 3(h), resp.). In order to assess the contribution of hNSSCs to CAM's neovasculature, immunohistochemical investigations of CAMs were undertaken and, as shown in Figure 4, the presence of a significant number of human cells within and in regions surrounding neovessels was observed. Cells positive for the following human angiogenic markers: vWF, CD31, SMA, and FXIIIa were observed, whereas the use of the human antibody against HLA-ABC indicated the engraftment of hNSSCs in chick CAM without notable cross reactivity with the chick cells (Figure 4). Concordant with data presented in Figure 3, CAMs transplanted with differentiated cells showed higher numbers of blood vessels compared to CAMs transplanted with undifferentiated hNSSCs. 

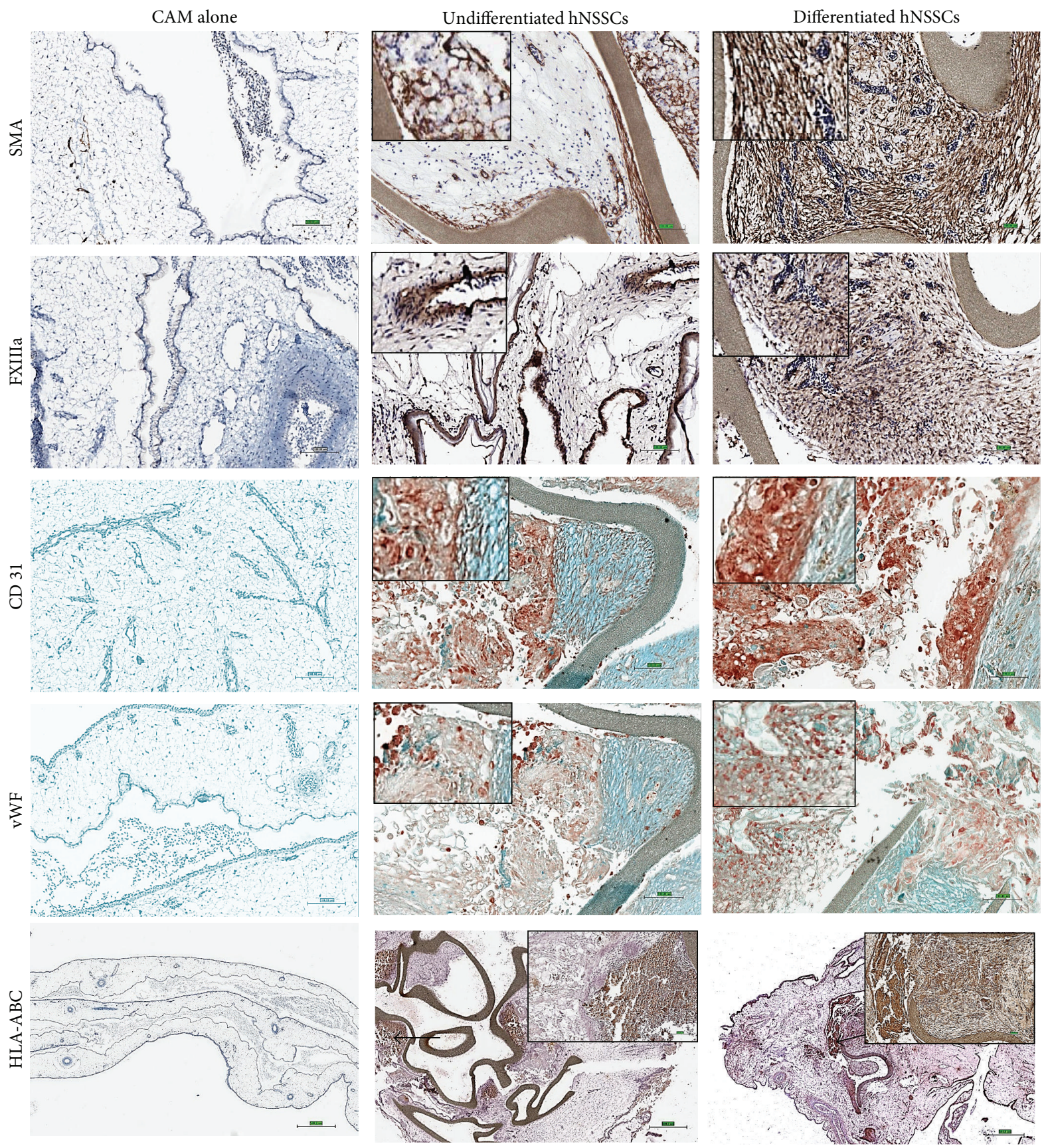

FIGURE 4: Immunohistochemical staining of CAM alone and CAM grafted with hNSSCs for endothelial-related markers. CAMs transplanted with undifferentiated and differentiated hNSSCs (10 days) were processed and stained using the indicated antibody. Brown colour indicates areas with positive staining for the respective cell marker (smooth muscle actin (SMA), factor XIIIa, CD31, and von Willebrand factor (vWF)). The human leukocyte antigen (HLA-ABC) was used to differentiate the engraftment of human cells from chick cells $(\mathrm{Bar}=100 \mu \mathrm{m})$.

3.4. CAMs Transplanted with Undifferentiated hNSSCs Exhibited Epidermal Tissue Formation Ex Vivo. CAMs transplanted with undifferentiated hNSSCs exhibited modest staining for angiogenic markers and a modest number of neovessels (Figure 4, middle panel). Interestingly, large numbers of transplanted undifferentiated hNSSCs were observed in association with epithelial-like structures with irregular vasculature and an epidermal like phenotype that stained positively for CD1a, CK5/6, CK19, and S-100 (Figure 5, middle panel). The chick and CAM were observed to be negative for the human CD1a, CK5/6, CK19, and S-100 further suggesting that undifferentiated hNSSCs displayed epidermal potential. Studying the epidermal potential of the hNSSCs population in depth will be the subject of an independent investigation. 

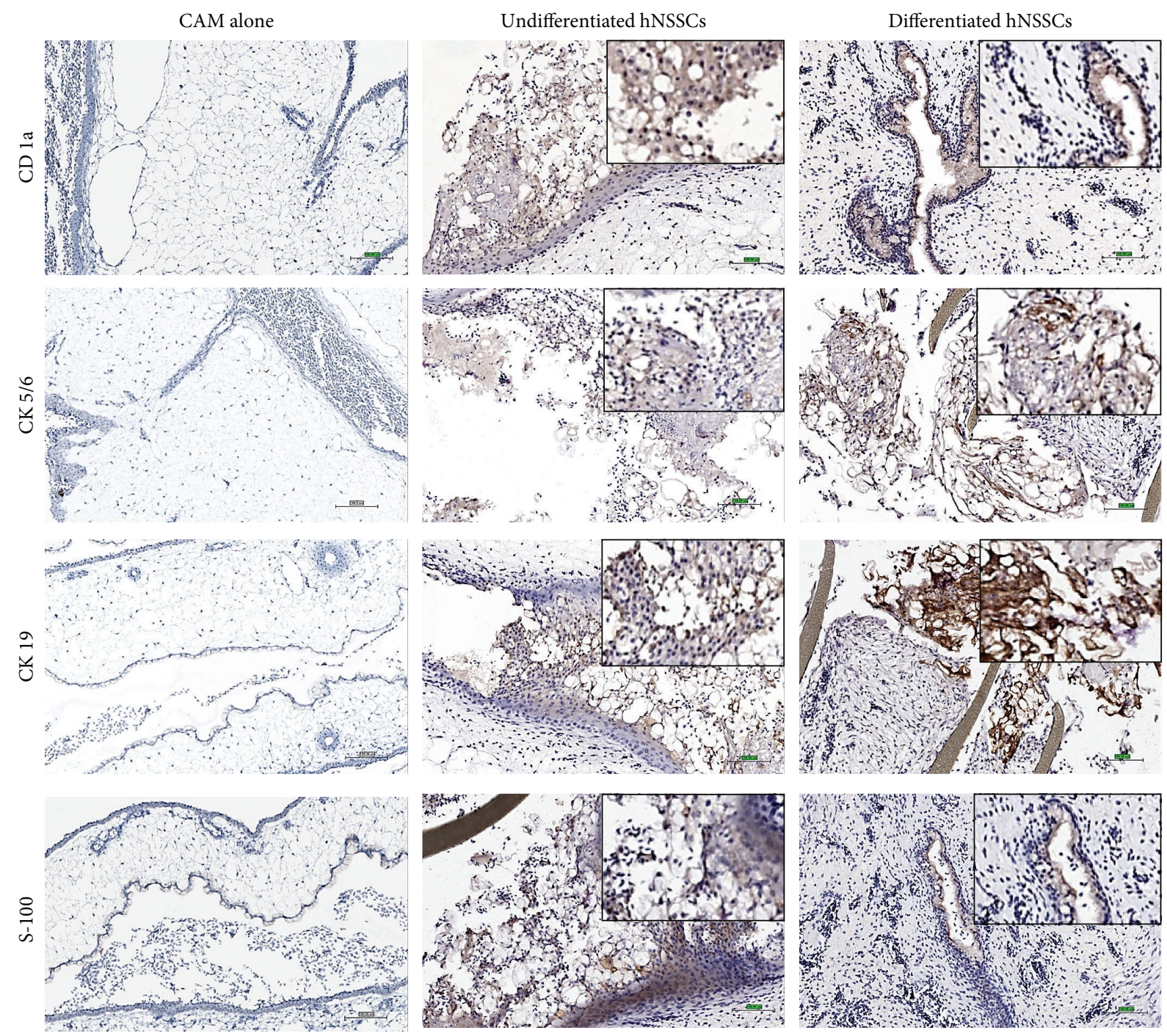

FIGURE 5: Immunohistochemical staining of CAM alone and CAM grafted hNSSCs for ectoderm-related markers. CAMs were grown as in Figure 4 and subsequently were processed for IHC. Brown colour indicates the positive area of respective skin lineage associated proteins, CD1a, CK 5/6, CK19, and S-100. CK: Cytokeratin $($ Bar $=100 \mu \mathrm{m})$.

\section{Discussion}

Angiogenesis plays pivotal role in a number of physiological and pathological conditions. Throughout adulthood, angiogenesis takes place in response to several physiological stimuli including hypoxia and inflammation, in wound healing and in tissue regeneration [20, 21]. Therefore, enhanced understanding of the molecular mechanisms and the cell types key in the modulation and regulation of angiogenesis is pivotal in a number of areas including tumour growth and regenerative medicine.

Neonatal skin stromal cells have recently emerged as a novel source of multipotent stem cells, with the potential to form a number of distinct lineages in vitro [22]. Thus neonatal skin derived $\mathrm{CD}_{13}{ }^{+} \mathrm{CD}_{3}{ }^{+} \mathrm{CD} 29^{+} \mathrm{CD} 44^{+} \mathrm{CD} 105^{+} \mathrm{CD} 0^{+}$ $\mathrm{CD} 45^{-} \mathrm{CD}^{-} 4^{-} \mathrm{CD}^{-} 1^{-} \mathrm{CD}^{-} 4^{-} \mathrm{HLADR}^{-}$stromal cells are capable of differentiating into endothelial cells and forming
$\mathrm{CD}^{+} 1^{+} \mathrm{VE}$ cadherin ${ }^{+} \mathrm{VEGF}^{+} \mathrm{vWF}^{+} \mathrm{eNOS}^{+}$capillary tubelike structures in in vitro $[8,22]$. However, understanding the precise contribution of hNSSCs to angiogenesis under ex vivo conditions remains unclear. The current study shows the potential of hNSSCs to aid the angiogenic process. The ALDH assay has been widely utilized as a functional marker to isolate cancer and normal stem cells and progenitor cells. ALDH positive cells were shown to be capable of self-renewal and to possess broad lineage differentiation potential $[18,19]$. In the current study, hNSSCs displayed significant numbers of ALDH ${ }^{\text {high }}$ cells, suggesting the presence of undifferentiated/progenitor cells. The application of an in vitro transwell migration and scratch assays demonstrated that hNSSCs possessed a high migration potential. The migratory potential of hNSSCs suggests additional roles for these cells in contributing to a number of repair mechanisms, including wound healing. 
The current study also suggests the potential of hNSSCs to differentiate along the endothelial lineage following placement ex vivo in an angiogenic niche, namely, the CAM. The CAM assay has long been established as a model for the study of tumor angiogenesis and metastasis, given the absence of a fully developed immune system in the chick at this early developmental stage [11]. In addition, the CAM is a robust, cost-effective facile model to enable scaffold, factor, and cell screening, providing an intermediate approach between simple in vitro and complex ex vivo approaches $[13,23]$. In this study, the presence of hNSSCs in neoangiogenesis in the CAM model was observed. Interestingly, extensive neoangiogenesis was observed with differentiated hNSSCs cultured under endothelial induction media prior to transplantation. The enhanced endothelial formation supports our earlier report of the angiogenic potential of hNSSCs in vitro [8]. Undifferentiated and differentiated hNSSCs were observed to augment angiogenesis in the CAM model. Cells positive for human vWF, CD31, SMA, and Factor XIIIa were observed in the endothelium of CAMs. Factor XIIIa has been shown to play a vital role in embryo implantation and to contribute to tissue remodelling and wound healing and to developmental processes that all involve angiogenesis. Factor XIIIa is regarded as a novel proangiogenic factor and has been shown to promote endothelial cell proliferation and migration and to inhibit apoptosis [24, 25]. Factor XIIIa has also been reported to be produced by multiple cell types including bone marrow derived cells and skin lineages. Factor XIIIa was observed in hypodermis of fetal skin at 6 weeks gestational ages and also in the subepidermal cellular network [26]. In the current study, we observed enhanced expression of Factor XIIIa stain in both undifferentiated and differentiated hNSSCs transplanted CAMs with increased intensity in CAMs transplanted with differentiated cells.

Differentiated hNSSCs exhibited an enhanced localization with the CAM blood vessel and were shown to exert a greater angiogenic effect than undifferentiated hNSSCs. In addition, although untested, the paracrine factors expressed by hNSSCs may also contribute to angiogenesis development, rather than direct differentiation into endothelial cells, though this hypothesis warrants further investigation.

Aside from that, undifferentiated hNSSCs exhibited epidermal differentiation capability as evidenced by CDla, CK5/6, CK19, and S-100. Interestingly, these markers were previously linked to dermal and epidermal cells types such as melanocytes, keratinocytes, Langerhans cells, and other epithelial cells $[27,28]$. However, these results require further in vivo validation to confirm if hNSSCs could find new application in areas such as wound healing.

\section{Conclusion}

The current study demonstrates for the first time the ability of hNSSCs to contribute to angiogenesis in an ex vivo system. Proliferation, differentiation, migration, cell-cell adhesion, cell-matrix interactions, and morphological regulation of endothelial cells are the important biological processes that drive vasculogenesis and angiogenesis. Our previous and current studies indicate that in vitro and ex vivo differentiation of
hNSSCs offers a useful resource to explore the mechanisms underlying cell biological regulation of angiogenesis. Our previous studies have shown, at least in vitro, that hNSSCs are multipotent, and the current study suggests the potential of those cells to differentiate into endothelial-like cells ex vivo. Thus, hNSSCs are a valuable cell resource that might be useful in applications requiring enhanced angiogenesis or in areas such as ischemic diseases. Furthermore, these cells could be employed in tissue engineering and cell based therapy in which vascularization is an essential component.

\section{Conflict of Interests}

The authors indicate no potential conflict of interests.

\section{Acknowledgments}

This project was funded by the National Plan for Science, Technology and Innovation (MAARIFAH), King Abdulaziz City for Science and technology, Kingdom of Saudi Arabia, Award no. 12-BIO3112-02. The authors thank Dr. Janos Kanczler and Mrs. Carol Roberts, Bone and Joint Research Group, Centre for Human Development Stem Cells and Regeneration, Institute of Developmental Science, University of Southampton Medical School, Southampton, UK, for providing technical support in this study. They thank Mr. Ali Al-Roalle, Department of Anatomy, College of Medicine, King Saud University, for histological study support.

\section{References}

[1] J. H. Kim, I. S. Park, Y. Park, Y. Jung, S. H. Kim, and S.-H. Kim, "Therapeutic angiogenesis of three-dimensionally cultured adipose-derived stem cells in rat infarcted hearts," Cytotherapy, vol. 15, no. 5, pp. 542-556, 2013.

[2] X. Wang, J. Ge, E. E. Tredget, and Y. Wu, “The mouse excisional wound splinting model, including applications for stem cell transplantation," Nature Protocols, vol. 8, no. 2, pp. 302-309, 2013.

[3] L. Wei, C. L. Keogh, V. R. Whitaker, M. H. Theus, and S. P. Yu, "Angiogenesis and stem cell transplantation as potential treatments of cerebral ischemic stroke," Pathophysiology, vol. 12, no. 1, pp. 47-63, 2005.

[4] M. F. Pittenger, A. M. Mackay, S. C. Beck et al., "Multilineage potential of adult human mesenchymal stem cells," Science, vol. 284, no. 5411, pp. 143-147, 1999.

[5] R. Vishnubalaji, M. Al-Nbaheen, B. Kadalmani, A. Aldahmash, and T. Ramesh, "Comparative investigation of the differentiation capability of bone-marrow- and adipose-derived mesenchymal stem cells by qualitative and quantitative analysis," Cell and Tissue Research, vol. 347, no. 2, pp. 419-427, 2012.

[6] M. Al-Nbaheen, R. vishnubalaji, D. Ali et al., "Human stromal (mesenchymal) stem cells from bone marrow, adipose tissue and skin exhibit differences in molecular phenotype and differentiation potential," Stem Cell Reviews and Reports, vol. 9, no. 1, pp. 32-43, 2013.

[7] D. Hamam, D. Ali, R. Vishnubalaji et al., "microRNA-320/ RUNX2 axis regulates adipocytic differentiation of human mesenchymal (skeletal) stem cells," Cell Death \& Disease, vol. 5, no. 10, Article ID e1499, 2014. 
[8] R. Vishnubalaji, M. Manikandan, M. Al-Nbaheen, B. Kadalmani, A. Aldahmash, and N. M. Alajez, "In vitro differentiation of human skin-derived multipotent stromal cells into putative endothelial-like cells," BMC Developmental Biology, vol. 12, article 7, 13 pages, 2012.

[9] A. Luethy, F. Stenner, C. Lohri et al., "Autologous stem cell transplantation: leukapheresis product has anti-angiogenic effects in vivo correlating with neutrophil-derived VEGFR1," Anticancer Research, vol. 31, no. 10, pp. 3115-3124, 2011.

[10] D. Ribatti, "Chicken chorioallantoic membrane angiogenesis model," Methods in Molecular Biology, vol. 843, pp. 47-57, 2012.

[11] D. Ribatti, "Chick embryo chorioallantoic membrane as a useful tool to study angiogenesis," International Review of Cell and Molecular Biology, vol. 270, pp. 181-224, 2008.

[12] J. M. Kanczler, E. L. Smith, C. A. Roberts, and R. O. C. Oreffo, "A novel approach for studying the temporal modulation of embryonic skeletal development using organotypic bone cultures and microcomputed tomography," Tissue EngineeringPart C: Methods, vol. 18, no. 10, pp. 747-760, 2012.

[13] E. L. Smith, J. M. Kanczler, and R. O. C. Oreffo, "A new take on an old story: chick limb organ culture for skeletal niche development and regenerative medicine evaluation," European Cells \& Materials, vol. 26, pp. 91-106, 2013.

[14] W. W. Kilarski, L. Petersson, P. F. Fuchs, M. S. Zielinski, and P. Gerwins, "An in vivo neovascularization assay for screening regulators of angiogenesis and assessing their effects on preexisting vessels," Angiogenesis, vol. 15, no. 4, pp. 643-655, 2012.

[15] N. A. Lokman, A. S. F. Elder, C. Ricciardelli, and M. K. Oehler, "Chick chorioallantoic membrane (CAM) assay as an in vivo model to study the effect of newly identified molecules on ovarian cancer invasion and metastasis," International Journal of Molecular Sciences, vol. 13, no. 8, pp. 9959-9970, 2012.

[16] M. Al-Toub, A. Almusa, M. Almajed et al., "Pleiotropic effects of cancer cells' secreted factors on human stromal (mesenchymal) stem cells," Stem Cell Research \& Therapy, vol. 4, no. 5, article 114, 2013.

[17] S. M. Irvine, J. Cayzer, E. M. Todd et al., "Quantification of in vitro and in vivo angiogenesis stimulated by ovine forestomach matrix biomaterial," Biomaterials, vol. 32, no. 27, pp. 6351-6361, 2011.

[18] C. Ginestier, M. H. Hur, E. Charafe-Jauffret et al., "ALDH1 is a marker of normal and malignant human mammary stem cells and a predictor of poor clinical outcome," Cell Stem Cell, vol. 1, no. 5, pp. 555-567, 2007.

[19] J. Douville, R. Beaulieu, and D. Balicki, "ALDH1 as a functional marker of cancer stem and progenitor cells," Stem Cells and Development, vol. 18, no. 1, pp. 17-26, 2009.

[20] S. Konisti, S. Kiriakidis, and E. M. Paleolog, "Hypoxia-a key regulator of angiogenesis and inflammation in rheumatoid arthritis," Nature Reviews Rheumatology, vol. 8, no. 3, pp. 153162, 2012.

[21] L. A. Dipietro, "Angiogenesis and scar formation in healing wounds," Current Opinion in Rheumatology, vol. 25, no. 1, pp. 87-91, 2013.

[22] R. Vishnubalaji, M. Al-Nbaheen, B. Kadalmani, A. Aldahmash, and T. Ramesh, "Skin-derived multipotent stromal cells-n archrival for mesenchymal stem cells," Cell and Tissue Research, vol. 350, no. 1, pp. 1-12, 2012.

[23] A. M. Cimpean, D. Ribatti, and M. Raica, "The chick embryo chorioallantoic membrane as a model to study tumor metastasis," Angiogenesis, vol. 11, no. 4, pp. 311-319, 2008.
[24] R. Dardik, A. Solomon, J. Loscalzo et al., "Novel proangiogenic effect of factor XIII associated with suppression of thrombospondin 1 expression," Arteriosclerosis, Thrombosis, and Vascular Biology, vol. 23, no. 8, pp. 1472-1477, 2003.

[25] S. M. Dallabrida, L. A. Falls, and D. H. Farrell, "Factor XIIIa supports microvascular endothelial cell adhesion and inhibits capillary tube formation in fibrin," Blood, vol. 95, no. 8, pp. 2586-2592, 2000.

[26] N. S. Gibran, B. J. Nickoloff, and K. A. Holbrook, "Ontogeny and characterization of factor XIIIa+ cells in developing human skin," Anatomy and Embryology, vol. 193, no. 1, pp. 35-41, 1996.

[27] T. Terada, "Pigmented adenoid cystic carcinoma of the ear skin arising from the epidermis: a case report with immunohistochemical studies," International Journal of Clinical and Experimental Pathology, vol. 5, no. 3, pp. 254-259, 2012.

[28] M. Cumberbatch, M. Singh, R. J. Dearman, H. S. Young, I. Kimber, and C. E. M. Griffiths, "Impaired Langerhans cell migration in psoriasis," The Journal of Experimental Medicine, vol. 203, no. 4, pp. 953-960, 2006. 

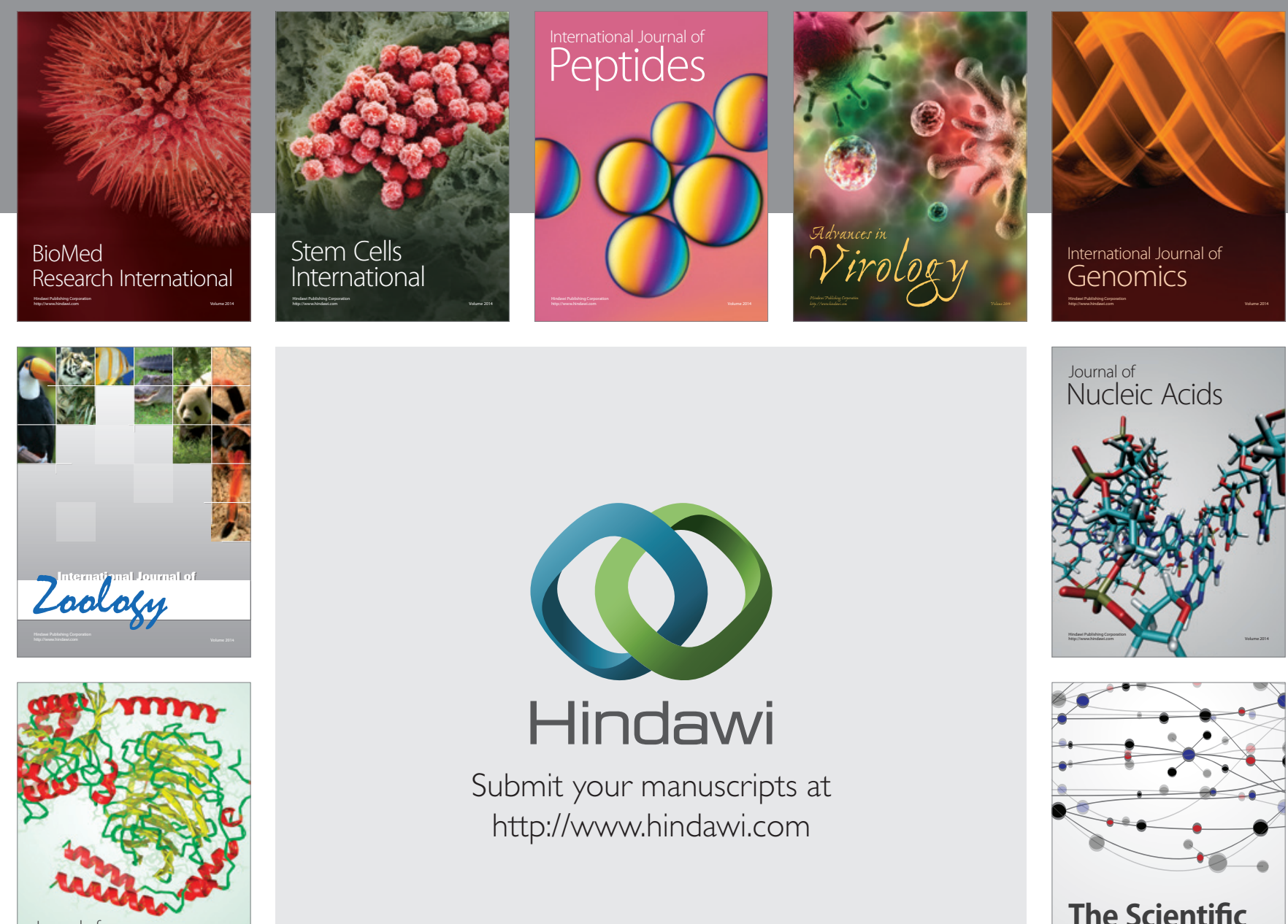

Submit your manuscripts at

http://www.hindawi.com

Journal of
Signal Transduction
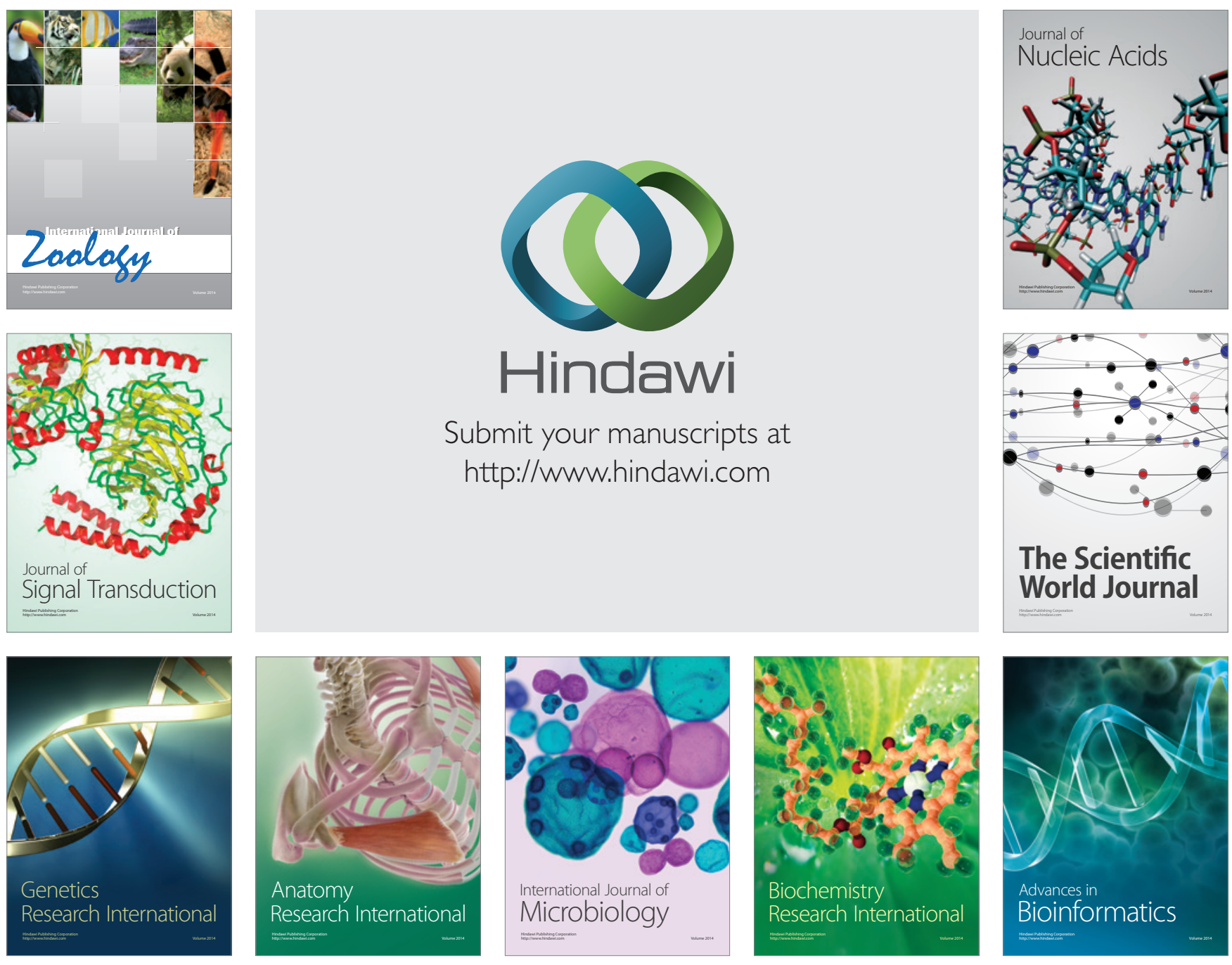

The Scientific World Journal
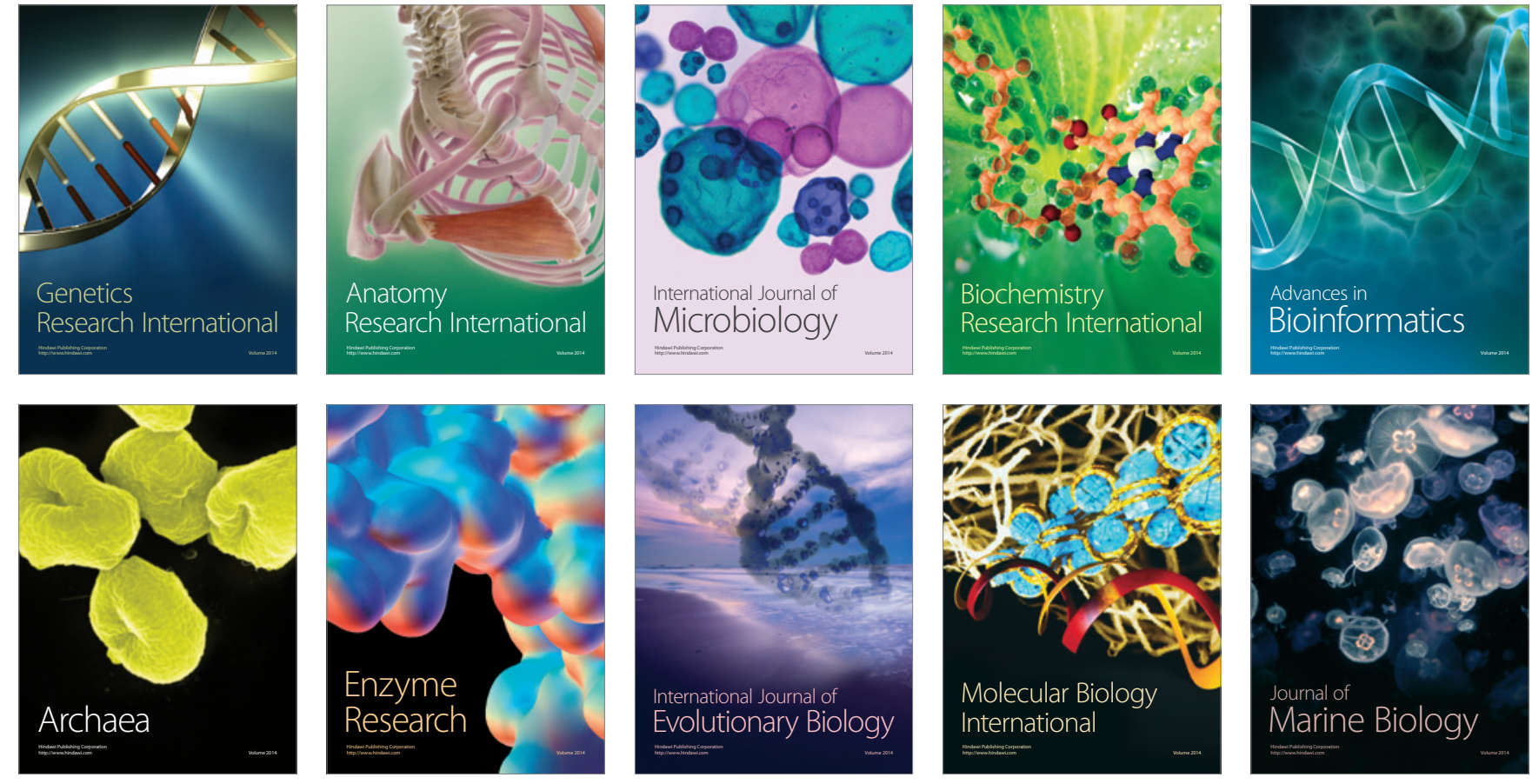